\title{
Does normouricemic status in acute gouty arthritis really reflect a normal status? Consider confounders of serum levels of urate
}

\author{
Hong Ki Min ${ }^{1}$ and Hae-Rim Kim²
}

${ }^{1}$ Division of Rheumatology, Department of Internal Medicine, Konkuk University Medical Center, Seoul; ${ }^{2}$ Division of Rheumatology, Department of Internal Medicine, Research Institute of Medical Science, Konkuk University School of Medicine, Seoul, Korea

Received: December 9, 2019

Accepted: December 11, 2019

\section{Correspondence to}

Hae-Rim Kim, M.D.

Division of Rheumatology,

Department of Internal Med-

icine, Research Institute of

Medical Science, Konkuk Uni-

versity School of Medicine, 120 -

1 Neungdong-ro, Gwangjin-gu,

Seoul 05030, Korea

Tel: +82-2-2030-7542

Fax: +82-2-2030-7728

E-mail: kimhaerim@kuh.ac.kr https://orcid.org/0000-0002-

1911-6236

\section{See Article on Page 240-248}

Gout is a form of inflammatory arthritis, the prevalence of which is increasing worldwide, including in Korea [1]. It is important to manage hyperuricemia to prevent recurrent flares, reduce tophal size, prevent erosion of bone, and suppress urate stone formation in the urinary tract. Furthermore, gout is associated with several chronic disorders including chronic kidney disease, coronary artery disease, heart failure, and metabolic syndrome. These associations are evident even in patients with asymptomatic hyperuricemia. To date, the serum level of urate is the best-known predictor of acute gouty arthritis flare [2]; this is why the American College of Rheumatology (ACR) and European League Against Rheumatism (EULAR) recommend to start urate lowering therapy (ULT) in patients with recurrent gout flare $[3,4]$. However, such arthritis may develop not only in hyperuricemic patients, but also when the urate level is normal or even below the lower reference limit. The recently updated EULAR guideline emphasizes that urate levels at initial diagnosis and during an acute flare are not sufficient to diagnose gout [5]. In addition, a sudden change in urate level may evoke acute gouty arthritis; both the ACR and EULAR guidelines recommend prophylactic colchicine for 6 months after initiation of ULT, and dose of ULT should be maintained during an acute flare $[3,4]$. Although the precise pathological mechanism of acute gouty arthritis developing after initial ULT remains unclear, the paradox is wellknown; rheumatologists know that hyperuricemia is not always evident in acute gouty arthritis patients.

In a recent issue of the Korean Journal of Internal Medicine, Lee et al. [6] focused on the differences between hyperuricemic and normouricemic acute gouty arthritis patients. Although the study was retrospective in nature and all patients were recruited from a single hospital, the characteristics of normouricemic acute gouty arthritis patients were described, as were factors triggering gout recurrence. Urano et al. [7] showed that a reduction in the urate level in acute gouty arthritis patients correlated with the levels of C-reactive protein (CRP) and interleukin-6, suggesting that an inflammatory response and increased urinary urate excretion explained the sudden decline in urate level. Lee et al. [6] reported similar results; normouricemic acute gouty arthritis patients exhibited higher-level inflammation in 
terms of higher white blood cell counts in blood and joint fluid, a higher CRP level, and more fever [6]. Thus, urate levels measured during an acute flare may be a useful indicator of severe gout.

Lee et al. [6] showed that several factors were associated with gout recurrence. Interestingly, the initial urate level was not, and females exhibited a significantly higher hazard ratio. However, Lin et al. [8] showed that the baseline urate level was the only predictor of gout development in asymptomatic hyperuricemic males; another study found that the risk for gout flare was higher in patients with baseline urate levels over than under $6 \mathrm{mg} / \mathrm{dL}$ [2]. Kim et al. [1] analyzed data in the Korean national health claims database and reported that gout was more prevalent in older males. An Italian group developed a Gout Activity Score (GAS) including urate levels, gout flare count over the past 12 months, and tophal size [9]. A higher GAS score, older age, and obesity predicted gout flare in the next 12 months, but females exhibited a lower incidence rate ratio [10]. Today, stepwise multivariate analyses are not recommended for clinical research [11], as the variables included are chosen only by reference to statistics. Errors can be made. Thus it is necessary to consider potential confounding or related other factors with results of stepwise multivariate analyses. Females become prone to gout only after menopause. Hence, whether older age and the female sex exhibit multicolinearity shoulde be considered in results of Lee et al. [6].

Many factors, including a dietary foods can affect urate levels [12]. Lee et al. [6] revealed that recent hemodialysis initiation and surgery were more common in a normouricemic than a hyperuricemic group $(9.1 \%$ vs. $2.3 \%, 22.7 \%$ vs. $6.0 \%$, respectively), these suggest that interpreting current serum urate level at acute flare needs careful review for potential confounder. Notably, gastrointestinal (GI) tract surgery tends to be more frequent in normouricemic patients ( $55 \%$ vs. $12.5 \%, p=0.088$ ) [6]. The GI tract is important both in terms of urate elimination (20\% to $30 \%$ of total excretion) and urate absorption from foods. The results of Lee et al. [6] suggest that a decrease in urate level caused by reduced absorption from food may be more marked than an increase in urate level caused by disturbance of urate excretion via the GI tract after surgery.

In normouricemia group, the serum urate level be- fore acute flare was significantly higher than urate level in acute flare state, and most of them (81.3\%) showed hyperuricemia before or after acute flare [6]. Thus, serum levels of urate during acute flare may not well represent the overall urate level, which is affected by many factors [12]. In diabetics, levels of hemoglobin Aic are useful because they reflect the average glucose level over the prior 8 to 12 weeks, greatly assisting physicians who must modulate medications. Novel laboratory tools representing a long-term, stable average value for serum level of urate would help physicians initiate or modify ULT.

In conclusion, serum levels of urate may be normal in some acute gouty arthritis patients, particularly those recently placed on hemodialysis or who have undergone surgery. Many factors influence the urate levels; careful interpretation and follow-up are required for acute gouty arthritis patients who are initially normouricemic.

\section{Conflict of interest}

No potential conflict of interest relevant to this article was reported.

\section{REFERENCES}

1. Kim JW, Kwak SG, Lee H, Kim SK, Choe JY, Park SH. Prevalence and incidence of gout in Korea: data from the national health claims database 2007-2015. Rheumatol Int 2017:37:1499-1506.

2. Halpern R, Fuldeore MJ, Mody RR, Patel PA, Mikuls TR. The effect of serum urate on gout flares and their associated costs: an administrative claims analysis. J Clin Rheumatol 2009;15:3-7.

3. Richette P, Doherty M, Pascual E, et al. 2016 Updated EULAR evidence-based recommendations for the management of gout. Ann Rheum Dis 2017;76:29-42.

4. Khanna D, Khanna PP, Fitzgerald JD, et al. 2012 American College of Rheumatology guidelines for management of gout. Part 2: therapy and antiinflammatory prophylaxis of acute gouty arthritis. Arthritis Care Res (Hoboken) 2012;64:1447-1461.

5. Richette P, Doherty M, Pascual E, et al. 2018 Updated European League Against Rheumatism evidencebased recommendations for the diagnosis of gout. Ann 
Rheum Dis 2019 Jun 5 [Epub]. https://doi.org/10.1136/ annrheumdis-2019-215315.

6. Lee JS, Kwon OC, Oh JS, et al. Clinical features and recurrent attack in gout patients according to serum urate levels during an acute attack. Korean J Intern Med 2019;35:240-248.

7. Urano $\mathrm{W}$, Yamanaka $\mathrm{H}$, Tsutani $\mathrm{H}$, et al. The inflammatory process in the mechanism of decreased serum uric acid concentrations during acute gouty arthritis. J Rheumatol 2002;29:1950-1953.

8. Lin KC, Lin HY, Chou P. The interaction between uric acid level and other risk factors on the development of gout among asymptomatic hyperuricemic men in a prospective study. J Rheumatol 2000;27:1501-1505.

9. Scire CA, Carrara G, Viroli C, et al. Development and first validation of a disease activity score for gout. Arthritis Care Res (Hoboken) 2016;68:1530-1537.

10. Chinchilla SP, Doherty M, Abhishek A. Gout Activity Score has predictive validity and is sensitive to change: results from the Nottingham Gout Treatment Trial (Phase II). Rheumatology (Oxford) 2019;5:1378-1382.

11. Lydersen S. Statistical review: frequently given comments. Ann Rheum Dis 2015;74:323-325.

12. de Oliveira EP, Burini RC. High plasma uric acid concentration: causes and consequences. Diabetol Metab Syndr 2012;4:12. 